\title{
Broad composite resonances and their signals at the $\mathrm{LHC}$
}

\author{
Da Liu, ${ }^{1, *}$ Lian-Tao Wang, ${ }^{2, \dagger}$ and Ke-Pan Xie ${ }^{3, \$}$ \\ ${ }^{1}$ High Energy Physics Division, Argonne National Laboratory, Argonne, Illinois 60439, USA \\ ${ }^{2}$ Department of Physics and Enrico Fermi Institute, The University of Chicago, \\ Chicago, Illinois 60637, USA \\ ${ }^{3}$ Center for Theoretical Physics, Department of Physics and Astronomy, Seoul National University, \\ Seoul 08826, Korea
}

(Received 21 January 2019; published 18 October 2019; corrected 6 October 2020)

\begin{abstract}
The existence of the $S U(2)_{L}$ triplet composite spin-1 resonances $\rho^{ \pm, 0}$ is a universal prediction of the strongly interacting new physics addressing the naturalness problem. Such resonances have not been found in the diboson final states, which are expected to be the dominant decay channels. In this work we propose a new scenario where the left-handed quark doublet $q_{L}=\left(t_{L}, b_{L}\right)$ is fully composite. In this case, the $\rho$ resonances can be broad and mainly decay to the third-generation quarks. The $t \bar{t}$ resonance search channel is comparable in sensitivity to the dilepton channel. In addition, the same-sign dilepton channel in $t \bar{t} \rho^{0}$-associated production can probe the large-width region and complement the Drell-Yan production channels.
\end{abstract}

DOI: 10.1103/PhysRevD.100.075021

\section{INTRODUCTION}

The discovery of a Higgs-like boson at the LHC [1,2] was a big step towards the understanding of the electroweak symmetry breaking (EWSB). An attractive solution to the associated naturalness problem is provided by the composite Higgs models, in which the Higgs is a pseudo-Nambu-Goldstone boson emerging from the spontaneous symmetry breaking $\mathcal{G} / \mathcal{H}$ of some strongly interacting composite sector at $\mathcal{O}(\mathrm{TeV})$. The EWSB is triggered by some explicit $\mathcal{G}$-breaking interactions between the elementary Standard Model (SM) sector and composite sector [3-5]. An important signal of composite Higgs models is the presence of composite resonances. A spin-1 resonance similar to the $\rho$ of QCD (denoted also as $\rho$ here) is probably one of the most obvious targets for collider searches. In most previous studies, the $\rho$ resonances tended to be narrow and decay dominantly to the SM diboson final states (i.e., $\left.W^{ \pm} Z / W^{ \pm} h, W^{+} W^{-} / Z h\right)[6,7]$. In this article, we propose a new scenario where the left-handed thirdgeneration quark doublet $q_{L}=\left(t_{L}, b_{L}\right)^{T}$ is a massless bound state from the composite sector. The $\rho$ resonances

\footnotetext{
*da.liu@anl.gov

†liantaow@uchicago.edu

kpxie@snu.ac.kr
}

Published by the American Physical Society under the terms of the Creative Commons Attribution 4.0 International license. Further distribution of this work must maintain attribution to the author(s) and the published article's title, journal citation, and DOI. Funded by SCOAP ${ }^{3}$. can be broad and mainly decay to the third-generation final states (i.e., $t \bar{t}, b \bar{b}, t \bar{b} / \bar{t} b$ ). The search for spin-1 composite resonances will be more challenging, as will be discussed in this work. Currently, the LHC experimental collaborations are planning for future search strategies, including the highluminosity upgrade. Our paper emphasizes a new direction: the search for broad resonances. We present a benchmark model in the framework of composite Higgs models, offering timely motivation and important targets for testing the strategies in this new direction of LHC searches.

The symmetry structure of a composite Higgs model is a coset $\mathcal{G} / \mathcal{H}$, where the strong dynamics preserves $\mathcal{H}$ even after confinement. Hence, the composite resonances should fill complete multiplets of $\mathcal{H}$. The SM gauge groups are embedded in $\mathcal{H}$. In popular benchmarks of the composite Higgs model, the SM fermions are treated as elementary. The third-generation fermions acquire their masses through mixing with composite fermions [3-5]. In this scenario, the coupling between the SM fermions and $\rho$ is suppressed by either $\rho$-SM gauge boson mixing or elementary-composite fermion mixing. The $\rho$ resonances couple strongly to other composite states, such as the longitudinal modes of $W$ and $Z$, and the Higgs boson. However, the lack of color factor enhancement and some accidental small factor lead to a decay width-mass ratio of the $\rho$ of $\mathcal{O}\left(g_{\rho}^{2} / 96 \pi\right)$. Hence, the $\rho$ resonance appears to be narrow even if $g_{\rho}$ is sizable.

The heaviness of the third-generation fermions motivates considering some of them as fully composite, as part of a complete multiplet of $\mathcal{H}$. Previously, $t_{R}$ has often been treated as a fully composite singlet of $\mathcal{H}[8,9]$. Though simple, this is not the only possibility. We will consider 
other possibilities. These give very different predictions for the width of the $\rho$ resonances, which lead to qualitatively new features and challenges for collider searches.

\section{THE MODEL}

We present here a model that realizes the new features discussed in the Introduction. Beginning with the frequently used coset $S O(5) / S O(4)$, we consider the lefthanded third-generation quarks as fully composite, embedded as a $\mathbf{4}$ of $S O(4)$,

$$
\Psi_{L}=\frac{1}{\sqrt{2}}\left(\begin{array}{c}
i b_{L}-i X_{L} \\
b_{L}+X_{L} \\
i t_{L}+i T_{L} \\
-t_{L}+T_{L}
\end{array}\right)_{2 / 3}=P\left(\begin{array}{c}
q_{L}^{X} \\
q_{L}
\end{array}\right),
$$

where $P$ is a $4 \times 4$ unitary matrix. Under the decomposition $S O(4) \times U(1)_{X} \rightarrow S U(2)_{L} \times U(1)_{Y}$, the quartet can be decomposed as bidoublets,

$$
\mathbf{4}_{2 / 3} \rightarrow \mathbf{2}_{7 / 6} \oplus \mathbf{2}_{1 / 6} .
$$

$q_{L}=\left(t_{L}, b_{L}\right)^{T}$ and $q_{L}^{X}=\left(X_{L}, T_{L}\right)^{T}$ have the SM quantum numbers $(\mathbf{3}, \mathbf{2})_{1 / 6}$ and $(\mathbf{3}, \mathbf{2})_{7 / 6}$, respectively. We assume that the right-handed top quark $t_{R}$ is elementary. We also introduce an elementary doublet $q_{R}^{X}=\left(X_{R}, T_{R}\right)^{T}$ with SM quantum number $(\mathbf{3}, \mathbf{2})_{7 / 6}$, which pairs with $q_{L}^{X}$ and becomes massive [10]. We will write them as incomplete 5's of $S O(5): t_{R}^{\mathbf{5}}=\left(0,0,0,0, t_{R}\right)_{2 / 3}^{T}$ and $q_{R}^{X 5}=$ $1 / \sqrt{2}\left(-i X_{R}, X_{R}, i T_{R}, T_{R}, 0\right)_{2 / 3}^{T}$. The spin- 1 composite resonances - the $\rho$ 's - span the adjoint of $S O(4)=S U(2)_{L} \times$ $S U(2)_{R}$. We mainly focus on the $\mathbf{3}$ of $S U(2)_{L}, \rho^{a_{L}}$, with $a=1,2,3$. The relevant Lagrangian, following the standard Callan-Coleman-Wess-Zumino procedure [11-13], is

$$
\begin{aligned}
\mathcal{L}= & -\frac{1}{4} \rho_{\mu \nu}^{a_{L}} \rho^{a_{L} \mu \nu}+\frac{m_{\rho}^{2}}{2 g_{\rho}^{2}}\left(g_{\rho} \rho_{\mu}^{a_{L}}-e_{\mu}^{a_{L}}\right)^{2}+\bar{q}_{R}^{X} i \not D q_{R}^{X} \\
& +\bar{t}_{R} i \not D t_{R}+\bar{\Psi}_{L} \gamma^{\mu}\left(i \nabla_{\mu}+\frac{2}{3} g_{1} B_{\mu}\right) \Psi_{L} \\
& +c_{1} \bar{\Psi}_{L} \gamma^{\mu} T^{a_{L}} \Psi_{L}\left(g_{\rho} \rho_{\mu}^{a_{L}}-e_{\mu}^{a_{L}}\right)-y_{1 R} f \bar{q}_{R}^{X 5} U \Psi_{L} \\
& -y_{2 R} f \bar{t}_{R}^{5} U \Psi_{L}+\text { H.c. }+\sum_{i} \alpha_{i} Q_{i},
\end{aligned}
$$

where $\nabla_{\mu}=\partial_{\mu}-i e_{\mu}^{a_{L}} T^{a_{L}}-i e_{\mu}^{a_{R}} T^{a_{R}}$. The field-strength tensor is $\rho_{\mu \nu}^{a_{L}}=\partial_{\mu} \rho_{\nu}^{a_{L}}-\partial_{\nu} \rho_{\mu}^{a_{L}}+g_{\rho} \epsilon^{a b c} \rho_{\mu}^{b_{L}} \rho_{\nu}^{c_{L}} . U=\exp \left\{i \frac{\sqrt{2}}{f} h_{i} T^{\hat{i}}\right\}$ is the Goldstone matrix, with $T^{\hat{i}}$ being the generators of $S O(5) / S O(4)$. The $Q_{i}$ 's are a set of higher-order operators [11]. The top-quark mass $M_{t}$ and the top partner masses $M_{T, X}$ are given by

$$
M_{t} \sim \frac{y_{2 R} v}{\sqrt{2}}, \quad M_{X}=y_{1 R} f, \quad M_{T} \sim y_{1 R} f .
$$

There are various indirect constraints on this model.

\section{THE ANOMALOUS COUPLINGS}

The first set of constraints comes from the modification of the $Z b_{L} \bar{b}_{L}, Z t_{L} \bar{t}_{L}$, and $W t_{L} \bar{b}_{L}$ couplings. The composite fermion kinetic term has an accidental $P_{L R}$ parity symmetry, which exchanges $T^{a_{L}} \leftrightarrow T^{a_{R}}$ and $e_{\mu}^{a_{L}} \leftrightarrow e_{\mu}^{a_{R}}[11,14]$. This protects the $Z b_{L} \bar{b}_{L}$ coupling at tree level, avoiding dangerous deviations with sizes $\xi \equiv v^{2} / f^{2}$. More explicitly, the Lagrangian in Eq. (3) contains two potentially dangerous operators: $\mathcal{O}_{L}^{q}=\bar{q}_{L} \gamma^{\mu} q_{L}\left(H^{\dagger} i \stackrel{\leftrightarrow}{D} \mu\right)$ and $\mathcal{O}_{L}^{(3) q}=$ $\bar{q}_{L} \gamma^{\mu} \sigma^{a} q_{L}\left(H^{\dagger} \sigma^{a} i \stackrel{\leftrightarrow}{D_{\mu}} H\right)$. They can modify the $Z b_{L} \bar{b}_{L}$ coupling as $\delta g_{L b}=-\frac{v^{2}}{2}\left(c_{L}^{q}+c_{L}^{(3) q}\right)$ [15]. In our model, these contributions cancel since $c_{L}^{q}=-c_{L}^{(3) q}=1 /\left(4 f^{2}\right)$, as shown in Eq. (3). However, the mass terms in Eq. (3) do not preserve the $P_{L R}$, which corrects the $Z b_{L} \bar{b}_{L}$ coupling at the one-loop level. We have checked that their constraints are weaker than the $S, T$-parameters $[16,17]$. The modifications to the $W t_{L} \bar{b}_{L}$ and $Z t_{L} \bar{t}_{L}$ couplings arise at tree level, given by $\delta g_{W t_{L} b_{L}} \sim \delta g_{Z t_{L} t_{L}} \sim-\xi / 4$ [18]. The bound from electroweak precision tests (EWPT) is $\left|\delta g_{Z t_{L} t_{L}}\right| \lesssim 8 \%$ [18,19], which limits $\xi \lesssim 0.32$. The newest limit of the $Z t_{L} \bar{t}_{L}$ coupling from $t \bar{t} Z$-associated production at the $36.1 \mathrm{fb}^{-1}$ LHC is $\left|\delta g_{Z t_{L} t_{L}}\right| \lesssim 10 \%$ (95\% C.L.) [20], corresponding to $\xi \lesssim 0.4$.

\section{OBLIQUE PARAMETERS}

In our model, the strong dynamics preserves an $S O(4)$ symmetry. Since $S O(4)$ contains the custodial $S U(2)$, there is no tree-level contribution to the $T$-parameter. The $S$-parameter receives a tree-level contribution from the mixing of $\rho$ and SM gauge bosons. One-loop contributions to $T$ and $S$ come from heavy quarks, $\rho$ resonances, and the modified Higgs-gauge boson couplings. Since our model in Eq. (3) is nonrenormalizable, the loop contributions to $S$ and $T$ are in principle incalculable. We regulate the divergence with a cutoff $\Lambda=4 \pi f$. We use the results of Refs. [21-23] for the contribution from the loop of $\rho$ 's, and calculate the contributions from fermion loops using the formulas in Ref. [24].

There can also be an additional contribution to $S$ parameter from higher-order operators. An operator that is particularly relevant is

$$
Q_{2}=g_{\rho} \rho_{\mu \nu}^{a_{L}} E^{a_{L} \mu \nu},
$$

where $E_{\mu \nu}^{a_{L}}$ is given in Ref. [25], and 
$E_{\mu \nu}^{a_{L}}=\cos ^{2} \frac{|\vec{h}|}{2 f} g_{2} W_{\mu \nu}^{a_{L}}-\frac{4}{|\vec{h}|^{2}} \sin ^{2} \frac{|\vec{h}|}{2 f} \vec{h}^{T} t^{a_{L}}\left(g_{1} B_{\mu \nu} t^{3_{R}}\right) \vec{h}$

in the unitary gauge. It can contribute to the mixing between $\rho$ and SM gauge bosons, and hence shift $S$ parameter. According to the so-called partial UV completion assumption, $\alpha_{2} \lesssim 1 / g_{\rho}^{2}$ [11]. Therefore, the kinetic mixing between $\rho_{\mu}$ and $W_{\mu}$ induced by $Q_{2}$ is subleading. We often define $\beta_{2}=g_{\rho}^{2} \alpha_{2}$, with $\beta_{2}$ being a $\mathcal{O}(1)$ parameter. There is no similar contribution to $T$-parameter, if we assume custodial symmetry is preserved in the UV completion. In Fig. 1 we plot the 95\% C.L. bound from $S$ and $T$ measurements in the $M_{\rho}-g_{\rho}$ plane with $a_{\rho}=m_{\rho} /\left(g_{\rho} f\right)=$ $1 / 2$ with different values for $\beta_{2}$ and $y_{1 R}$ (see the Appendix for the analytical formulas for $S$ and $T$ in our model at leading order in $\xi$ ), using the limits on $S$ and $T$ in Ref. [26]:

$$
S=0.02 \pm 0.07, \quad T=0.06 \pm 0.06,
$$

with a strong correlation of $92 \%$. Note that the mass of the top partner is roughly given by $y_{1 R} f$. A small $y_{1 R}$ will lead to a strong constraint on $f$ due to contributions to $S$ and $T$ of $\mathcal{O}\left(M_{t}^{2} / M_{T}^{2}\right)$. Meanwhile, $y_{1 R}$ explicitly breaks the custodial symmetry and a larger $y_{1 R}$ will lead to a stronger constraint. We find that $y_{1 R} \sim 2$ gives the weakest bound in our parameter space, as can be seen from the figure. $\beta_{2}=1 / 4$ significantly relaxes bounds in the small- $M_{\rho}$ region, as the tree-level contributions to $S$-parameter from the higher-dimensional operator $Q_{2}$ and the mass term cancel. The bounds from EWPT can be further relaxed if there is a new positive contribution to $T$-parameter $[21,22,27-29]$.

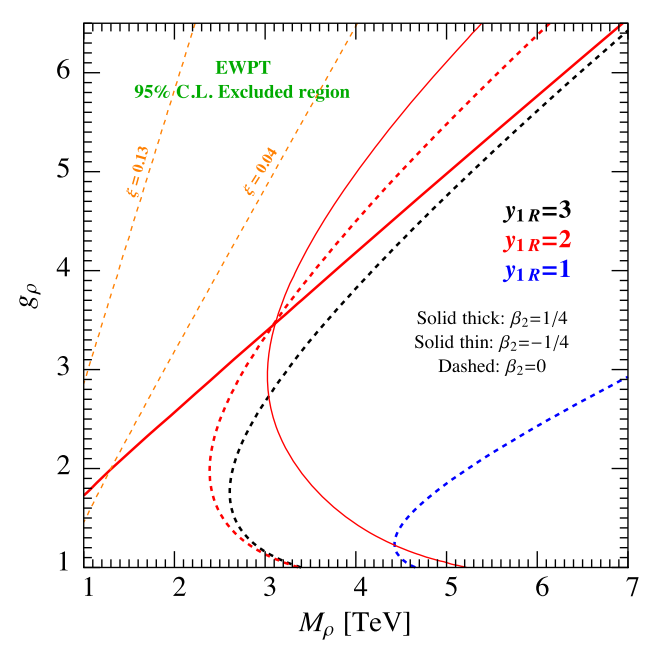

FIG. 1. The indirect bound (95\% C.L.) from EWPT in the $M_{\rho}-g_{\rho}$ plane for $a_{\rho}=1 / 2$ with different values for $y_{1 R}$ and $\beta_{2}$. The orange contours $\xi=0.13$ and 0.04 represent the indirect bounds at the current and $3 \mathrm{ab}^{-1}$ LHC [30-34], respectively.
Finally, we briefly comment on the flavor physics implications. The main issue is the mass difference between the $B$ and $\bar{B}$ mesons, which requires [35]

$$
\xi c_{4 q}\left(\frac{\theta_{b d}}{V_{u b}}\right)^{2}<2 \times 10^{-3}
$$

where $\theta_{b d}$ is the projection of $b_{L}$ into the $d$ mass eigenstate, and $c_{4 q}$ is the Wilson coefficient of the operator $\left(1 / f^{2}\right) \bar{q}_{L} \gamma^{\mu} q_{L} \bar{q}_{L} \gamma_{\mu} q_{L}$. In general, this strongly constrains the fully composite left-handed top-quark scenario [36,37]. However, this constraint is very dependent on the underlying theory of flavor. For example, the CabibboKobayashi-Maskawa matrix can originate from the up-type quark sector. In this case, the down-type sector is flavor diagonal, i.e., $\theta_{b d}=0$, making the model free of the $B$-physics constraint.

\section{COLLIDER SIGNAL}

The most significant difference between the collider signal of the spin-1 composite resonances in our model and those of the previously used benchmarks is the width. The branching ratios into different final states and the total decay width for the neutral resonance $\rho^{0}$ are shown in Fig. 2. Since $q_{L}$ is fully composite, its coupling to $\rho$ is of the order $g_{\rho}$. The dominant decay channels are $t \bar{t}$ and $b \bar{b}$ for $M_{\rho}<2 M_{X}$. If $M_{\rho}>2 M_{X}$, the decay into top partner pair is significant, which is almost half of the total decay widths in this region. Broad $\rho$ resonances caused by the decay to top partners were studied in Refs. [38,39]. The branching ratio to the diboson final state is suppressed by a factor of $a_{\rho}^{4} /\left(2 N_{c}\right)$. The suppression of the diboson branching ratio, especially at small $a_{\rho}$, makes them much less relevant.

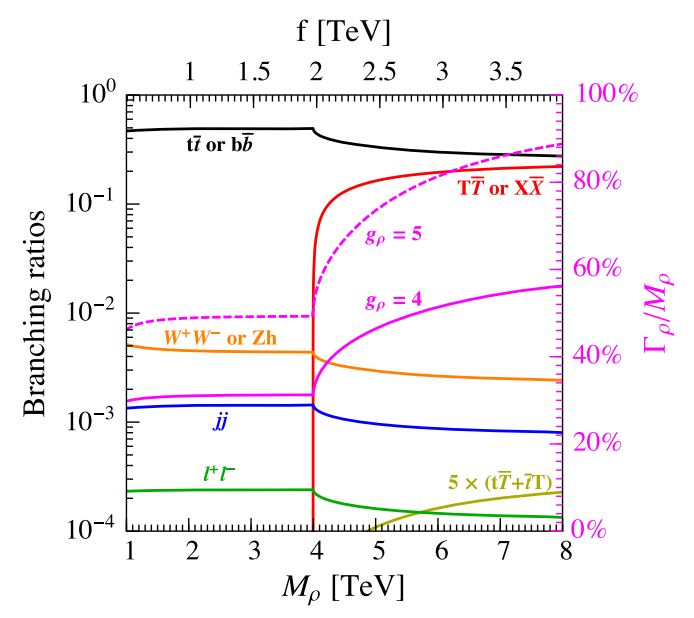

FIG. 2. The decay branching ratios and the total width-mass ratios of $\rho$. We set $g_{\rho}=4, a_{\rho}=1 / 2, M_{X}=2 \mathrm{TeV}$, and $c_{1}=1$ for the branching ratios. For $\Gamma_{\rho} / M_{\rho}$, the two magenta curves represent $g_{\rho}=4$ (solid) and 5 (dashed). 


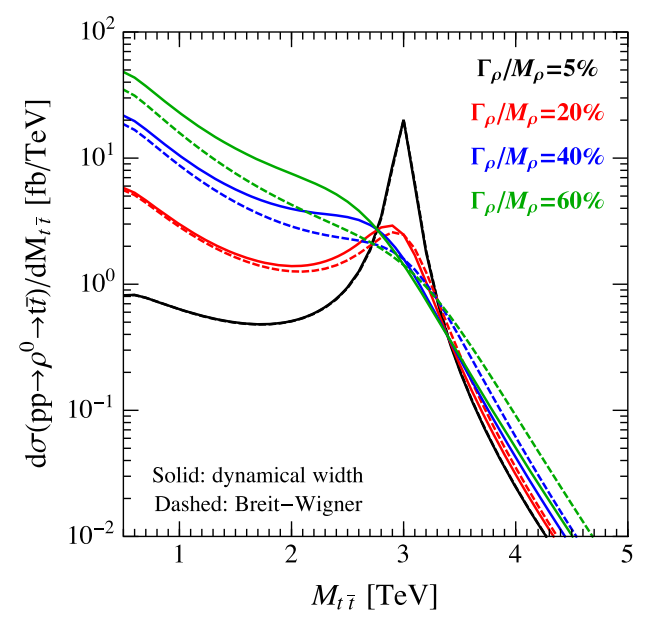

FIG. 3. Shape of the broad composite resonance at the LHC. We set $M_{\rho}=3 \mathrm{TeV}, a_{\rho}=1 / 2, y_{1 R}=2$, and $c_{1}=1$.

This is very different from the well-studied cases, where the diboson channel is the most sensitive [7].

For broad resonances, the usual narrow-width approximation does not apply, nor is it correct to just add a large constant width to the propagator. Instead, we need to replace the propagator

$$
\frac{1}{\left(\hat{s}-M_{\rho}^{2}\right)^{2}+M_{\rho}^{2} \Gamma_{\rho}^{2}} \rightarrow \frac{1}{\left(\hat{s}-M_{\rho}^{2}\right)^{2}+\hat{s}^{2} \Gamma_{\rho}^{2} / M_{\rho}^{2}},
$$

where $\sqrt{\hat{s}}$ is the parton center-of-mass energy. This has a significant impact on the shape of the resonance at the LHC, as shown in Fig. 3.

There is no LHC search fully optimized for the broad resonances presented here. Achieving maximal sensitivity will be a challenge that deserves much more detailed studies. In the following, we will recast some of the LHC searches that still have sensitivity and highlight the difference with the well-studied benchmarks. ${ }^{1}$ First of all, the searches in the diboson channel are not sensitive due to its suppressed branching ratio. Moreover, the limit set by searching for narrow resonances in the $t \bar{t}, b \bar{b}, t \bar{b} / \bar{t} b$, and $\ell^{+} \ell^{-}$final states will not apply if $\Gamma_{\rho} / M_{\rho}>40 \%$. The systematic uncertainties on the backgrounds will have a large impact for the large-width case. There are several broad resonance searches at the LHC in the above channels, but most of the searches have used the constant decay width approximation which could mismodel the signal. For the $t \bar{t}$ channel, the large-width effect has been considered up to $\Gamma_{\rho} / M_{\rho} \sim 30 \%$ by ATLAS [41] and CMS [42]. While ATLAS searched in the semileptonic final state, the CMS analysis combined all possible final states and is more sensitive. In Fig. 4, we show the present limits and

\footnotetext{
${ }^{1}$ Another dedicated recasting for a similar scenario can be found in a recent study [40].
}

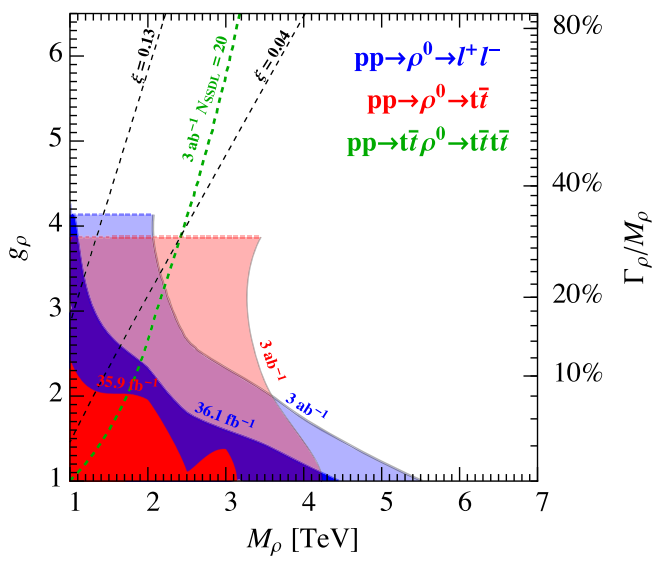

FIG. 4. The current and projected constraints. The parameter benchmarks are $y_{1 R}=2, a_{\rho}=1 / 2$, and $c_{1}=1$. The $t \bar{t}$ and $\ell^{+} \ell^{-}$bounds are based on the results from CMS [42] and ATLAS [43], respectively. For the $t \bar{t} \rho^{0} \rightarrow t \bar{t} t \bar{t}$ channel, we use the SSDL event contour $N\left(\ell^{ \pm} \ell^{ \pm}+\right.$jets $)=20$ to set an estimate for the $3 \mathrm{ab}^{-1} \mathrm{LHC}$.

projected $\left(3 \mathrm{ab}^{-1}\right)$ reach for the $t \bar{t}$ channel (red shaded region) based on the CMS result. The colored regions are truncated at $g_{\rho} \sim 4\left(\Gamma_{\rho} / M_{\rho} \leq 30 \%\right)$, beyond which reliable extrapolations from current searches are not possible. When $g_{\rho}$ increases, the reach of $M_{\rho}$ first decreases because of the suppression of the coupling between the $\rho$ resonance and valence quarks at large $g_{\rho}$. It then increases as the $b \bar{b}$ initiated production becomes important. The possibility of a broad $\rho^{0}$ decaying into $\ell^{+} \ell^{-}$has been studied by ATLAS [43], up to $\Gamma_{\rho} / M_{\rho}=32 \%$. The corresponding limit and its extrapolation to $3 \mathrm{ab}^{-1}$ are shown in Fig. 4 (blue regions). The mass reach in the low- $g_{\rho}$ region is higher than the $t \bar{t}$ channel, while in the high- $g_{\rho}$ region, due to the branching ratio suppression, $\ell^{+} \ell^{-}$is worse.

Currently, there is no strong constraint from the $b \bar{b}$ channel. ATLAS has searched for a broad $b \bar{b}$ resonance up to $\Gamma_{\rho} / M_{\rho}=15 \%$ [44], but the constraint is too weak to be shown in Fig. 4 due to the low integrated luminosity $\left(3.2 \mathrm{fb}^{-1}\right)$. CMS searched in the dijet channel for both the narrow and broad resonances [45]. The study considered the dynamical width effect, and gave results for $\Gamma_{\rho} / M_{\rho}$ up to $30 \%$. Without $b$ tagging in this search, its limit is weak.

Besides the Drell-Yan processes, there are other sensitive channels. Since the left-handed top is strongly coupled, the same-sign dilepton (SSDL) channel in the four-top final state $p p \rightarrow t \bar{t} \rho^{0} \rightarrow t \bar{t} t \bar{t}$ can be useful [46]. This channel has a mild dependence on the modeling of the width. The estimated sensitivity in our parameter space set by requiring 20 SSDL signal events (the green contour) is shown in Fig. 4. This channel can cover the large- $g_{\rho}$ region, which is hard to probe via Drell-Yan processes.

The signature in the large-coupling region $g_{\rho} \gtrsim 4$ would be a very broad heavy resonance in the $\ell^{+} \ell^{-}, t \bar{t}, b \bar{b}$, 
and $t \bar{b} / \bar{t} b$ final states. One possible way to enhance the sensitivity is to consider the interference between the signal and the SM irreducible background. This is similar to exploring the energy-increasing behavior coming from the higher-dimensional (four fermion) operators [47-56]. Since our $\rho$ resonance is color neutral, the Drell-Yan production channel does not interfere with the QCD $t \bar{t}$ background. The $t$-channel $b b \rightarrow b b$ and Drell-Yan $\ell^{+} \ell^{-}$channels do interfere with the SM irreducible backgrounds. Due to the suppression of the bottom parton distribution function at high energy and the suppression of the dilepton branching ratio at large $g_{\rho}$, they do not have significant sensitivity to the region $M_{\rho} \gtrsim 4 \mathrm{TeV}, g_{\rho} \gtrsim 4$. The productions of top partners $T$ and $X$ can probe our model. Compared to pair production, the single production of $T$ and $X$ can reach a higher mass region. We estimate the sensitivity in the SSDL channel from the single production of the charge-5/3 top partner $X$, requiring $N\left(\ell^{ \pm} \ell^{ \pm}+\right.$jets $)=20$. It can reach $M_{X} \sim 2.6 \mathrm{TeV}$ (corresponding to $\xi \sim 0.036$, with $y_{1 R}=2$, $a_{\rho}=1 / 2$, and $c_{1}=1$ ). Additional handles on the signal could become important as the reconstruction of a sharp resonance is less effective. For example, the $\rho$ resonance strongly interacts with the left-handed top quarks, and the polarization measurement of the top quarks in the $t \bar{t}$ final states can also help improve the sensitivity. It is well known that in the top-quark rest frame, the polar angle $\theta_{\ell}^{*}$ distribution of the charged lepton from the decay $t \rightarrow b \ell^{+} \nu$ reflects the right- (left-)handed polarization of the top quark, i.e., $d N / d \cos \theta_{\ell}^{*} \sim 1 \pm$ $\cos \theta_{\ell}^{*}$ [57-62]. Since the QCD-produced top pairs are unpolarized, this distribution asymmetry can be used to distinguish our signal from the background. See, e.g., Refs. [63,64] for detailed studies.

\section{CONCLUSION}

In this article we considered the scenario that the lefthanded third-generation quark doublet $q_{L}=\left(t_{L}, b_{L}\right)^{T}$ is a massless bound state of the strong dynamics, using the minimal coset $S O(5) / S O(4)$ as an example. We studied the constraints on our model from EWPT $(S, T$-parameters, and $\delta g_{L b}$ ) and direct searches at the LHC. Instead of the diboson final state in the case of narrow spin-1 resonances in the minimal composite Higgs model, the smoking gun signature of our model is the broad resonances in the $t \bar{t}, b \bar{b}$, $\ell^{+} \ell^{-}, t \bar{b} / \bar{t} b$, and four-top final state channels. We have recast the searches at the present LHC and made projections for the HL-LHC. We found that $t \bar{t}$ is comparable to the dilepton channel in our model, and the SSDL from the fourtop channel can probe the large- $g_{\rho}$ region. Further studies, taking into account additional information such as the top angular distribution and polarization, are needed to fully optimize the search for such broad composite resonances.

\section{ACKNOWLEDGMENTS}

We would like to thank Roberto Contino and Jiayin $\mathrm{Gu}$ for useful discussions. L. T. W. is supported by the DOE Grant No. DE-SC0013642. D. L. is supported in part by the U.S. Department of Energy under Contract No. DEAC02-06CH11357. K. P.X. is supported in part by the National Research Foundation of Korea under Grant No. 2017R1D1A1B03030820.

\section{APPENDIX: ANALYTICAL FORMULAS FOR $S, T$-PARAMETERS}

In this Appendix, we list the analytical formulas for $S, T$ parameters in our model. We assume that $\xi$ is small and keep the leading terms in the $\xi$ expansion. As discussed in the main text, the total contribution can be divided into three classes: the fermion loop, the $\rho$ resonance loop, and the Higgs loop with a modified Higgs-gauge boson coupling:

$$
S=S_{f}+S_{\rho}+S_{H}, \quad T=T_{f}+T_{\rho}+T_{H} .
$$

The result for $S$-parameter reads

$$
\begin{aligned}
S_{f}= & -\frac{N_{c} M_{t}^{2}\left(4 \ln \left(\frac{y_{1 f^{2}} f^{2}}{M_{t}^{2}}\right)-15\right)}{18 \pi y_{1 R}^{2} f^{2}} \\
& -\frac{N_{c} \xi\left(4 \ln \left(\frac{M_{t} y_{1 R}^{5} f^{5}}{\mu^{6}}\right)+17-12 \ln \frac{\Lambda^{2}}{M_{t}^{2}}\right)}{36 \pi}, \\
S_{\rho}= & \frac{4 \pi \xi}{g_{\rho}^{2}}\left(1-4 \beta_{2}\right)-\frac{\xi}{6 \pi}\left[1+\frac{41}{16} a_{\rho}^{2}\right. \\
& +\frac{3}{4}\left(a_{\rho}^{2}+28+24 \beta_{2}\left(a_{\rho}^{2} \beta_{2}-a_{\rho}^{2}-2\right)\right) \log \frac{\Lambda}{m_{\rho}} \\
& \left.-\frac{3}{2} \beta_{2}\left(9 a_{\rho}^{2}-4\right)+\frac{3}{2} \beta_{2}^{2}\left(9 a_{\rho}^{2}-8\right)\right], \\
S_{H}= & \frac{\xi}{12 \pi} \ln \frac{\Lambda^{2}}{M_{h}^{2}},
\end{aligned}
$$

while the result for $T$-parameter reads

$$
\begin{aligned}
T_{f}= & -\frac{N_{c} M_{t}^{4}\left(6 \ln \left(\frac{y_{1 R}^{2} f^{2}}{M_{t}^{2}}\right)-11\right)}{24 \pi M_{W}^{2} s_{W}^{2} y_{1 R}^{2} f^{2}} \\
& +\frac{N_{c} M_{t}^{2} \xi\left(\frac{3}{2} \ln \left(\frac{y_{1 R}^{2} f^{2}}{M_{t}^{2}}\right)-5\right)}{24 \pi M_{W}^{2} s_{W}^{2}}-\frac{N_{c} y_{1 R}^{2} v^{2} \xi}{96 \pi M_{W}^{2} s_{W}^{2}}, \\
T_{\rho}= & \frac{9 \xi}{32 \pi c_{W}^{2}} a_{\rho}^{2}\left[\left(1-\frac{8}{3} \beta_{2}^{2}\right) \log \frac{\Lambda}{m_{\rho}}+\frac{3}{4}-\frac{4}{3} \beta_{2}+\frac{2}{9} \beta_{2}^{2}\right], \\
T_{H}= & -\frac{3}{16 \pi} \frac{\xi}{c_{W}^{2}} \ln \frac{\Lambda^{2}}{M_{h}^{2}} .
\end{aligned}
$$


The cutoff $\Lambda$ is chosen as $4 \pi f$. From the formulas, we can see that the IR contributions to $S$ and $T$ from the modified Higgs-gauge boson coupling are anticorrelated. Since the measurements of $S, T$-parameters are strongly correlated (92\%), this will put a strong bound on $\xi \lesssim 0.012$, if there are no other contributions. In our model, both $S_{f}$ and $T_{f}$ tend to be negative and the absolute value of $T_{f}$ is preferred to be larger than $S_{f}$. Since the $\rho$ contribution can be positive, adding the $\rho$ contribution can relax the bound a little bit.
[1] G. Aad et al. (ATLAS Collaboration), Phys. Lett. B 716, 1 (2012).

[2] S. Chatrchyan et al. (CMS Collaboration), Phys. Lett. B 716, 30 (2012).

[3] D. B. Kaplan, Nucl. Phys. B365, 259 (1991).

[4] R. Contino, Y. Nomura, and A. Pomarol, Nucl. Phys. B671, 148 (2003).

[5] K. Agashe, R. Contino, and A. Pomarol, Nucl. Phys. B719, 165 (2005).

[6] A. Thamm, R. Torre, and A. Wulzer, Phys. Rev. Lett. 115, 221802 (2015).

[7] D. Pappadopulo, A. Thamm, R. Torre, and A. Wulzer, J. High Energy Phys. 09 (2014) 060.

[8] A. De Simone, O. Matsedonskyi, R. Rattazzi, and A. Wulzer, J. High Energy Phys. 04 (2013) 004.

[9] D. Marzocca, M. Serone, and J. Shu, J. High Energy Phys. 08 (2012) 013.

[10] K. Agashe, R. Contino, and R. Sundrum, Phys. Rev. Lett. 95, 171804 (2005).

[11] R. Contino, D. Marzocca, D. Pappadopulo, and R. Rattazzi, J. High Energy Phys. 10 (2011) 081.

[12] D. Greco and D. Liu, J. High Energy Phys. 12 (2014) 126.

[13] D. Liu, L.-T. Wang, and K.-P. Xie, J. High Energy Phys. 01 (2019) 157.

[14] K. Agashe, R. Contino, L. Da Rold, and A. Pomarol, Phys. Lett. B 641, 62 (2006).

[15] S. Gori, J. Gu, and L.-T. Wang, J. High Energy Phys. 04 (2016) 062.

[16] G. Panico, M. Safari, and M. Serone, J. High Energy Phys. 02 (2011) 103.

[17] C. Delaunay, O. Gedalia, S. J. Lee, G. Perez, and E. Ponton, Phys. Rev. D 83, 115003 (2011).

[18] A. Efrati, A. Falkowski, and Y. Soreq, J. High Energy Phys. 07 (2015) 018.

[19] G. Panico, in 10th International Workshop on Top Quark Physics (TOP2017) Braga, Portugal, 2017 (2018) [arXiv: 1801.03882].

[20] ATLAS Collaboration, CERN Technical Report No. ATLAS-CONF-2018-047, 2018.

[21] D. Ghosh, M. Salvarezza, and F. Senia, Nucl. Phys. B914, 346 (2017).

[22] R. Contino and M. Salvarezza, J. High Energy Phys. 07 (2015) 065.

[23] R. Contino and M. Salvarezza, Phys. Rev. D 92, 115010 (2015).

[24] L. Lavoura and J. P. Silva, Phys. Rev. D 47, 2046 (1993).

[25] G. Panico and A. Wulzer, Lect. Notes Phys. 913, 1 (2016).
[26] M. Tanabashi et al. (Particle Data Group), Phys. Rev. D 98, 030001 (2018).

[27] J. de Blas, M. Ciuchini, E. Franco, S. Mishima, M. Pierini, L. Reina, and L. Silvestrini, Proc. Sci., ICHEP2016 (2017) 690.

[28] M. Ciuchini, E. Franco, S. Mishima, M. Pierini, L. Reina, and L. Silvestrini, Nucl. Part. Phys. Proc. 273-275, 2219 (2016).

[29] M. Ciuchini, E. Franco, S. Mishima, and L. Silvestrini, J. High Energy Phys. 08 (2013) 106.

[30] V. Sanz and J. Setford, Adv. High Energy Phys. 2018, 7168480 (2018).

[31] J. de Blas, O. Eberhardt, and C. Krause, J. High Energy Phys. 07 (2018) 048.

[32] CMS Collaboration, CERN Technical Reports No. CMSNOTE-2012-006 and No. CERN-CMS-NOTE-2012-006, 2012.

[33] CMS Collaboration, CERN Technical Report No. ATLPHYS-PUB-2013-014, 2013.

[34] S. Dawson et al., in 2013 Community Summer Study on the Future of U.S. Particle Physics (Springer, Berlin Heidelberg, 2013).

[35] G. F. Giudice, C. Grojean, A. Pomarol, and R. Rattazzi, J. High Energy Phys. 06 (2007) 045.

[36] M. Gillioz, Phys. Rev. D 80, 055003 (2009).

[37] R. Barbieri, B. Bellazzini, V. S. Rychkov, and A. Varagnolo, Phys. Rev. D 76, 115008 (2007).

[38] D. Barducci, A. Belyaev, S. De Curtis, S. Moretti, and G. M. Pruna, J. High Energy Phys. 04 (2013) 152.

[39] D. Barducci and C. Delaunay, J. High Energy Phys. 02 (2016) 055.

[40] M. Gintner and J. Juráň, arXiv:1908.11619.

[41] M. Aaboud et al. (ATLAS Collaboration), Eur. Phys. J. C 78, 565 (2018).

[42] A. M. Sirunyan et al. (CMS Collaboration), J. High Energy Phys. 04 (2019) 031.

[43] M. Aaboud et al. (ATLAS Collaboration), J. High Energy Phys. 10 (2017) 182.

[44] M. Aaboud et al. (ATLAS Collaboration), Phys. Lett. B 759, 229 (2016).

[45] A. M. Sirunyan et al. (CMS Collaboration), J. High Energy Phys. 08 (2018) 130.

[46] D. Liu and R. Mahbubani, J. High Energy Phys. 04 (2016) 116.

[47] R. Franceschini, G. Panico, A. Pomarol, F. Riva, and A. Wulzer, J. High Energy Phys. 02 (2018) 111.

[48] D. Liu and L.-T. Wang, Phys. Rev. D 99, 055001 (2019). 
[49] S. Alioli, M. Farina, D. Pappadopulo, and J. T. Ruderman, Phys. Rev. Lett. 120, 101801 (2018).

[50] S. Alioli, M. Farina, D. Pappadopulo, and J. T. Ruderman, J. High Energy Phys. 07 (2017) 097.

[51] M. Farina, G. Panico, D. Pappadopulo, J. T. Ruderman, R. Torre, and A. Wulzer, Phys. Lett. B 772, 210 (2017).

[52] O. Domenech, A. Pomarol, and J. Serra, Phys. Rev. D 85, 074030 (2012).

[53] A. Pomarol and J. Serra, Phys. Rev. D 78, 074026 (2008).

[54] B. Bellazzini, F. Riva, J. Serra, and F. Sgarlata, J. High Energy Phys. 11 (2017) 020.

[55] R. Kelley, L. Randall, and B. Shuve, J. High Energy Phys. 02 (2011) 014.

[56] S. Banerjee, C. Englert, R. S. Gupta, and M. Spannowsky, Phys. Rev. D 98, 095012 (2018).

[57] B. Grzadkowski and Z. Hioki, Phys. Lett. B 529, 82 (2002).
[58] B. Grzadkowski and Z. Hioki, Phys. Lett. B 557, 55 (2003).

[59] R. M. Godbole, S. D. Rindani, and R. K. Singh, J. High Energy Phys. 12 (2006) 021.

[60] R. M. Godbole, S. D. Rindani, K. Rao, and R. K. Singh, AIP Conf. Proc. 1200, 682 (2010).

[61] R. M. Godbole, K. Rao, S. D. Rindani, and R. K. Singh, J. High Energy Phys. 11 (2010) 144.

[62] R. M. Godbole, M. E. Peskin, S. D. Rindani, and R. K. Singh, Phys. Lett. B 790, 322 (2019).

[63] K. Agashe, A. Belyaev, T. Krupovnickas, G. Perez, and J. Virzi, Phys. Rev. D 77, 015003 (2008).

[64] L. Cerrito, D. Millar, S. Moretti, and F. Span, arXiv: 1609.05540.

Correction: A misspelling in an author name in Ref. [40] has been fixed. 